\title{
Ionizing radiation-induced microRNA expression changes in cultured RGC-5 cells
}

\author{
KAIJUN WANG ${ }^{1,2}$, MEIJUAN ZHU ${ }^{1,2}$, PANPAN YE ${ }^{1,2}$, GUODI CHEN $^{3}$, WEI WANG ${ }^{1,2}$ and MIN CHEN MAN $^{1,2}$ \\ ${ }^{1}$ Eye Center; ${ }^{2}$ Zhejiang Provincial Key Laboratory of Ophthalmology; ${ }^{3}$ Department of Radiation Oncology, \\ The Second Affiliated Hospital, Medical College of Zhejiang University, Hangzhou, Zhejiang 310009, P.R. China
}

Received September 6, 2014; Accepted May 27, 2015

DOI: $10.3892 / \mathrm{mmr} .2015 .3938$

\begin{abstract}
MicroRNAs (miRNAs) are a class of short non-coding RNAs that regulate gene expression at the post-transcriptional level. It has been demonstrated that miRNAs serve a crucial role in tissue development and the pathogenesis of numerous diseases. The aim of the current study was to investigate the alterations in miRNA expression in a cultured retinal ganglion cell line (RGC-5 cells) following ionizing radiation injury. Cultured RGC-5 cells were exposed to X-rays at doses of 2, 4, 6 and 8 Gy using a medical linear accelerator. Alterations in cellular morphology were observed under a phase contrast microscope and cell viability was measured using the MTT assay. Subsequent to exposure to $\mathrm{X}$-ray radiation for 5 days, the viability of RGC- 5 cells was significantly reduced in the 6 and 8 Gy groups, accompanied by morphological alterations. Total RNA was then extracted from RGC-5 cells and subjected to miRNA microarray analysis subsequent to exposure to $6 \mathrm{~Gy} \mathrm{X}$-ray radiation for 5 days. The results of the microarray analysis indicated that the expression levels of 12 miRNAs were significantly different between the $6 \mathrm{~Gy}$ and control groups, including 6 upregulated miRNAs and 6 downregulated miRNAs. To verify microarray results, a reverse transcription-quantitative polymerase chain reaction (RT-qPCR) analysis was performed. The data obtained from RT-qPCR analysis was similar to that of the the microarray analysis for alterations in the expression of the 12 miRNAs. The results of the current study indicated that miRNA expression was sensitive to ionizing radiation, which may serve an important role in mechanisms of radiation injury in retinal ganglion cells.
\end{abstract}

Correspondence to: Dr Min Chen, Eye Center, The Second Affiliated Hospital, Medical College of Zhejiang University, 88 Jiefang Road, Hangzhou, Zhejiang 310009, P.R. China

E-mail: sisi5460@126.com

Key words: ionizing radiation, microRNAs, retinal ganglion cell

\section{Introduction}

MicroRNAs (miRNAs) are a class of short non-coding RNAs that regulate gene expression predominantly at the post-transcriptional level. At present, almost 2,000 unique mature human miRNAs have been identified, which serve critical roles in tissue development and the pathogenesis of numerous diseases (1-3). Greater than 250 miRNAs have been reported to be expressed in the retina (4). It has been demonstrated that miRNA-mediated gene regulation is a major mechanism underlying retinal and optic nerve development, function and associated diseases (5).

Radiation-induced retinopathy and radiation-induced optic neuropathy (RION) are severe complications of radiotherapy for intracranial, skull-base and sinus tumors, which may result in irreversible visual loss. Approximately $85 \%$ of affected eyes have final visual acuity of $6 / 60$ or worse (6). However, the detailed molecular mechanisms of radiation-induced retinopathy and RION remain unclear. Previous studies have demonstrated that miRNA expression is sensitive to ionizing radiation injury and alterations in miRNA expression appear to be important in the mechanisms of radiation-induced injury and associated disease $(7,8)$.

Although radiation-induced retinopathy and RION have been extensively investigated, the clinical therapeutic options remain limited (9). According to the characteristics of these diseases, early diagnosis is extremely important for prevention and treatment of RION $(10,11)$. It has been reported that miRNAs can serve as improved biomarkers for the early diagnosis of numerous diseases, including cancer (12), autoimmune diseases (13), cardiovascular diseases (14), ectopic pregnancy (15) and systemic inflammatory response syndrome (16). In contrast to other biomarkers, miRNAs are protected from endogenous RNase activity and are highly stable. For this reason, they have been considered as ideal biomarkers for use in early diagnosis, prediction of prognosis and therapeutic management (17).

The current study aimed to screen for altered expression levels of miRNAs in RGC-5 cells exposed to ionizing radiation, in order to identify the possible mechanisms of these altered miRNAs in radiation injury, and to explore the possibility of using specific miRNAs as potential biomarkers for radiation-induced retinopathy and RION. 


\section{Materials and methods}

RGC-5 cell culture. The RGC-5 cell line (American Type Culture Collection, Manassas, VA, USA) was grown in modified RPMI 1640 medium (Gibco Life Technologies, Carlsbad, CA, USA), containing $10 \%$ fetal bovine serum (Gibco Life Technologies) and $1 \%$ penicillin-streptomycin $(100 \mathrm{U} / \mathrm{ml}$ penicillin and $100 \mu \mathrm{g} / \mathrm{ml}$ streptomycin; Sigma-Aldrich, St. Louis, $\mathrm{MO}, \mathrm{USA})$ at $37^{\circ} \mathrm{C}$ and $5 \% \mathrm{CO}_{2}$. Cells were usually passaged at a ratio of 1:20. During cultivation, RGC-5 cells exhibited the same morphological phenotype in different generations. For viability assays, cells were plated onto positively charged 96-well plates. For microarray and reverse transcription-quantitative polymerase chain reaction (RT-qPCR) analysis, cells were harvested from $25 \mathrm{~cm}^{2}$ filter-capped cell culture flasks.

Radiation treatment and cell viability assay. The Siemens Medical Linear Accelerator (6MV Oncor; Siemens, Munich, Germany) was used in the current study to mimic radiation injury in vitro. RGC-5 cells were irradiated with $250 \mathrm{cGy} / \mathrm{min}$ dose rate X-rays at final doses of 2, 4, 6 and $8 \mathrm{~Gy}$. Unexposed cells served as controls. Following radiation, cells were cultured in a humidified incubator for 5 days and morphological alterations were observed daily using a phase-contrast microscope (Eclipse 90i; Nikon, Tokyo, Japan). Cell viability was evaluated by an MTT assay according to the manufacturer's instructions. Briefly, $20 \mu 1$ of $5 \mathrm{mg} / \mathrm{ml}$ MTT dye (Sigma-Aldrich) was added to each well and cells were incubated at $37^{\circ} \mathrm{C}$ for $4 \mathrm{~h}$. Subsequently, the supernatant was removed and purple-colored precipitates of formazan were dissolved by gently shaking for 10 min in $150 \mu \mathrm{l}$ dimethyl sulfoxide (Sangon Biotech Co., Ltd., Shanghai, China). The microplate was read at $570 \mathrm{~nm}$ (Benchmark Microplate Reader; Bio-Rad Laboratories, Inc., Hercules, CA, USA). The optical density (A value) of each sample was measured and applied for cell viability calculation.

RNA extraction and microarray assay. Total RNA of RGC-5 cells was extracted and purified using the mirVana ${ }^{\mathrm{TM}}$ miRNA Isolation kit (Ambion Life Technologies, Austin, TX, USA) according to the manufacturer's instructions, then were checked for a RIN number to inspect RNA integration by an Agilent Bioanalyzer 2100 (Agilent Technologies, Inc., Santa Clara, CA, USA). To analyze miRNA expression, total RNA samples $(100 \mathrm{ng})$ containing miRNAs were labeled with cyanine $3-\mathrm{pCp}$ (Agilent Technologies, Inc.) using the Agilent miRNAs Complete Labeling and Hyb kit (Agilent Technologies, Inc.). Slides were then hybridized for $20 \mathrm{~h}$ at $55^{\circ} \mathrm{C}$ using a hybridization system (G2535A; Agilent Technologies, Inc.). Subsequent to hybridization, the slides were washed in Agilent GE Wash Buffer 1 with Triton X-102, followed by Agilent GE Wash Buffer 2 with Triton X-102. All slides were immediately scanned using the Agilent Microarray Scanner and Feature Extraction software, version 10.7 (Agilent Technologies, Inc.) with the default settings. Raw data were normalized by the Quantile algorithm with Gene Spring Software, version 11.0 (Agilent Technologies, Inc.).

$R T-q P C R$. The expression levels of miRNAs were confirmed with SYBR-based quantitative PCR (RT-qPCR). Briefly, a total of $1 \mu \mathrm{g}$ RNA from each sample was transcribed into cDNA
Table I. Primer sequences used for the miRNA RT-qPCR analysis.

\begin{tabular}{ll} 
miRNAs & \multicolumn{1}{c}{ Primer sequence (5'-3') } \\
\hline U6 & AAGGATGACACGCAAATTCG \\
rno-miR-192 & CTGACCTATGAATTGACAGCC \\
rno-miR-210 & CTGTGCGTGTGACAGCGGCTGA \\
rno-miR-212 & TAACAGTCTCCAGTCACGGCCA \\
rno-miR-219-5p & TGATTGTCCAAACGCAATTCT \\
rno-miR-22 & AAGCTGCCAGTTGAAGAACTGT \\
rno-miR-338 & TCCAGCATCAGTGATTTTGTTGA \\
rno-miR-34a & TGGCAGTGTCTTAGCTGGTTGT \\
rno-miR-34a* & AATCAGCAAGTATACTGCCCTA \\
rno-miR-34c & AGGCAGTGTAGTTAGCTGATTGC \\
rno-miR-503 & TAGCAGCGGGAACAGTACTGCAG \\
rno-miR-505* & GGGAGCCAGGAAGTATTGATGTT \\
rno-miR-877 & GTAGAGGAGATGGCGCAGGG
\end{tabular}

RT-qPCR, reverse transcription-quantitative polymerase chain reaction; $\mathrm{miR}$, microRNA.

using the PrimeScript RT reagent kit (GeneCopoeia, Inc., Guangzhou, China) according to the manufacturer's instructions. The RT product $(10 \mu \mathrm{l})$ was diluted with $\mathrm{H}_{2} \mathrm{O}$ up to $50 \mu \mathrm{l}$. cDNA was then amplified by PCR with primers specific to the target sequence (Table I). Amplification conditions were as follows: $60 \mathrm{~min}$ incubation at $37^{\circ} \mathrm{C}$, followed by a $5 \mathrm{~min}$ termination reaction at $85^{\circ} \mathrm{C}$. Dilutions of cDNA in the PCR were adjusted for each gene with the aim of remaining within the linear range of amplification. RT-qPCR was performed with SYBR Green Realtime PCR Master mix (GeneCopoeia, Inc.) according to the manufacturer's instructions. Cycling conditions were as follows: Initial denaturation for $10 \mathrm{~min}$ at $95^{\circ} \mathrm{C}$, 40 cycles of melting $\left(95^{\circ} \mathrm{C}\right.$ for $\left.20 \mathrm{sec}\right)$, annealing $\left(62.5^{\circ} \mathrm{C}\right.$ for $20 \mathrm{sec})$ and extending $\left(72^{\circ} \mathrm{C}\right.$ for $\left.20 \mathrm{sec}\right)$. The relative alterations in expression for each miRNA were calculated by the cycle threshold method ( $\Delta \Delta \mathrm{Ct}$ method) as described previously (17).

Statistical analysis. Statistical analysis was performed with SPSS software, version 11.3 (SPSS, Inc., Chicago, IL, USA). For all assays, experiments were performed a minimum of three times. Data are presented as the mean \pm standard deviation and were evaluated by one-way analysis of variance and Student's t-test. $\mathrm{P}<0.05$ was considered to indicate a statistically significant difference.

\section{Results}

Effect of radiation on RGC-5 cell morphology and viability. As demonstrated by phase contrast microscopy, the majority of cells exhibited abnormal appearance in the 6 and 8 Gy groups following 5 days exposure to X-ray radiation, with the densities reduced and the cell shapes changing from a normal appearance to rounded and swollen. Compared with the control group, no significant morphological alterations were observed in the 2 and 4 Gy groups (Fig. 1A-E). 
A

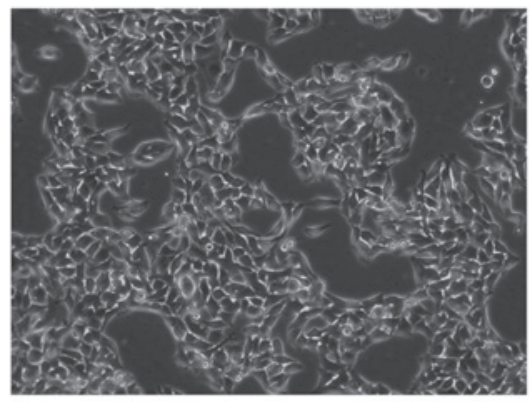

C

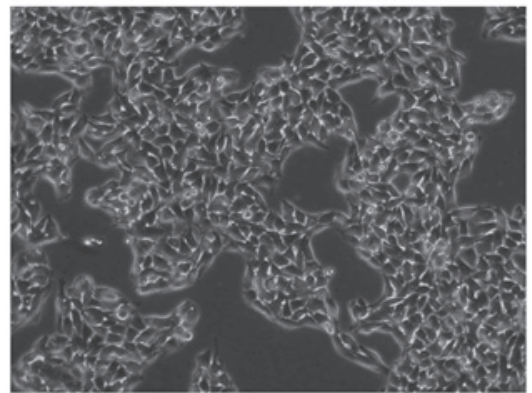

E

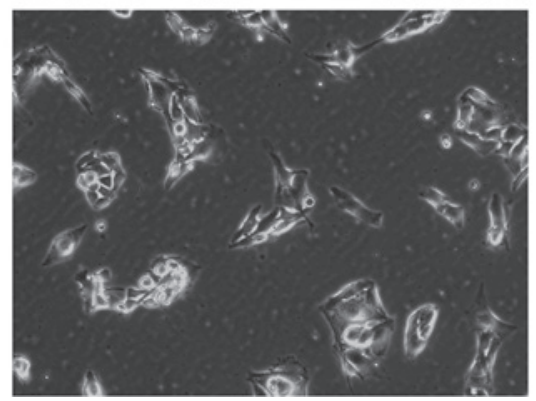

B

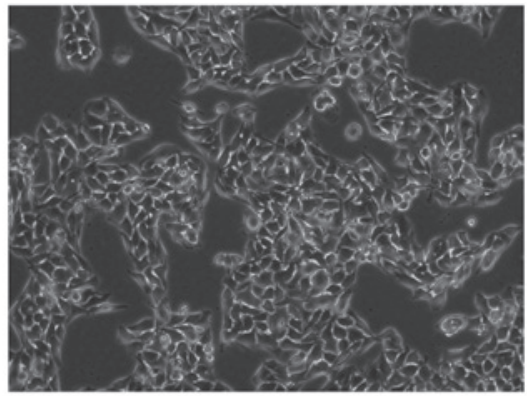

D

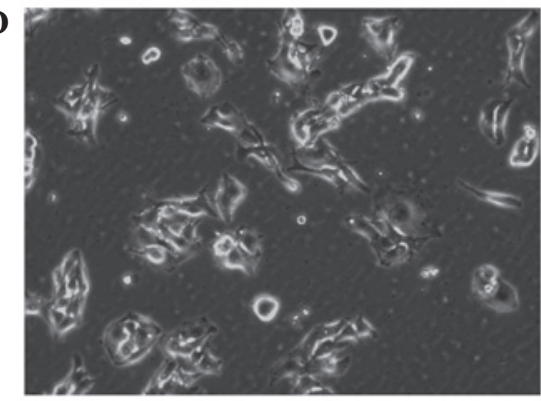

F

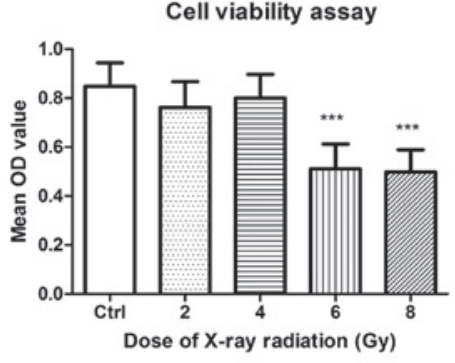

Figure 1. Morphological alterations and cell viability analysis of RGC-5 cells subsequent to exposure to different doses of radiation for 5 days, magnification x100. (A) The control group. (B) Cells treated with 2 Gy radiation. (C) Cells treated with 4 Gy radiation. There were no significant alterations in the morphology of the cells in group B or C, compared with the control group. (D) 6 Gy radiation group. The density of cells significantly reduced compared with the control group. Rounding and swelling of RGC-5 cells was observed under the phase-contrast microscope. (E) 8 Gy radiation group. The density and shape of cells were similar to the 6 Gy group. (F) An MTT assay demonstrated that the cell viability significantly reduced in 6 Gy and 8 Gy groups compared with the control group $\left({ }^{* * *} \mathrm{P}<0.01, \mathrm{n}=4\right)$. There was no statistical significance between $6 \mathrm{~Gy}$ and 8 Gy groups $(\mathrm{P}>0.05)$. Cell viability of 2 Gy and $4 \mathrm{~Gy}$ groups did not change compared with the control group ( $\mathrm{P}>0.05$, one-way analysis of variance). OD, optical density.

The MTT assay (Fig. 1F) indicated that the cell viability was significantly reduced in the 6 and 8 Gy groups following 5 days of radiation treatment $(\mathrm{P}<0.01)$. However, there was no significance difference between the 6 and 8 Gy groups $(\mathrm{P}>0.05)$. No significant differences in cell viability of the 2 and 4 Gy groups were identified compared with the control group $(\mathrm{P}>0.05)$, which was consistent with the morphological alterations described above.

Alterations in miRNA expression following radiation injury. According to the results of the MTT assay, the 6 Gy dose of radiation was applied in RGC-5 cells to evaluate miRNA expression. In total, 677 miRNA probes were included in the miRNA microarray. Subsequent to exposure to 6 Gy radiation for 5 days, the expression levels of 37 miRNAs were statistically altered compared with the control group $(\mathrm{P}<0.05)$, with 16 upregulated miRNAs and 21 downregulated miRNAs. Among these altered miRNAs, 12 were selected according to the fold change $(>1.5$ or $<0.667)$ and microarray repeatability (stable expression in triplicate experiments) (Fig. 2; repeated 3 times). Of these 12 miRNAs, 6 were upregulated [miRNA-192 (miR-192), -34a*, -877, -34c, -34a and -22; $\mathrm{P}<0.05$ and fold change $>1.5$ ] and 6 were downregulated
(miR-212, $-338,-219-5 \mathrm{p},-503,-210$ and $-505^{*} ; \mathrm{P}<0.05$ and fold change $<0.667$ ) (Table II).

Independent $R T-q P C R$ analysis to confirm microarray results. In order to validate microarray data, RT-qPCR analysis of miRNA expression was further performed for all of the 12 significantly altered miRNAs. The detailed RT-qPCR results compared with the results of microarray analysis (described as fold change) are presented in Table II. The expression levels of the 12 miRNAs exhibited similar alterations when measured by RT-qPCR $(\mathrm{P}<0.05)$, consistent with the results of microarray analysis.

\section{Discussion}

miRNAs have been extensively investigated and have been recognized to act as critical regulators of gene expression by binding to their targets. miRNAs have revolutionized the understanding of gene regulatory networks, providing novel tools to manage the development and clinical therapy of numerous diseases. It has been demonstrated that miRNAs are essential mediators and serve key roles in retinal ganglion cell axon branching and optic nerve formation $(18,19)$. However, 
Table II. Microarray and RT-qPCR results of miRNA expression in RGC-5 cells subsequent to exposure to 6 Gy radiation for 5 days (fold changes).

\begin{tabular}{lcc}
\hline miRNA name & Microarray & RT-qPCR \\
\hline Upregulated & & \\
miR-192 & 72.75 & $1.76^{\mathrm{b}}$ \\
miR-34a* & 46.13 & $2.10^{\mathrm{b}}$ \\
miR-877 & 6.97 & $1.68^{\mathrm{b}}$ \\
miR-34c & 2.71 & $1.70^{\mathrm{b}}$ \\
miR-34a & 1.84 & $2.26^{\mathrm{b}}$ \\
miR-22 & 1.82 & $1.96^{\mathrm{b}}$ \\
Downregulated & & \\
miR-212 & 0.66 & $0.38^{\mathrm{a}}$ \\
miR-338 & 0.64 & $0.48^{\mathrm{b}}$ \\
miR-219-5p & 0.50 & $0.41^{\mathrm{a}}$ \\
miR-503 & 0.38 & $0.65^{\mathrm{b}}$ \\
miR-210 & 0.28 & $0.58^{\mathrm{b}}$ \\
miR-505 & 0.02 & $0.26^{\mathrm{b}}$ \\
\hline
\end{tabular}

${ }^{\mathrm{a}} \mathrm{P}<0.05,{ }^{\mathrm{b}} \mathrm{P}<0.01$, fold change of microarray vs. RT-qPCR, (Student's t-test, $n=3)$. RT-qPCR, reverse transcription-quantitative polymerase chain reaction; miRNA, microRNA.

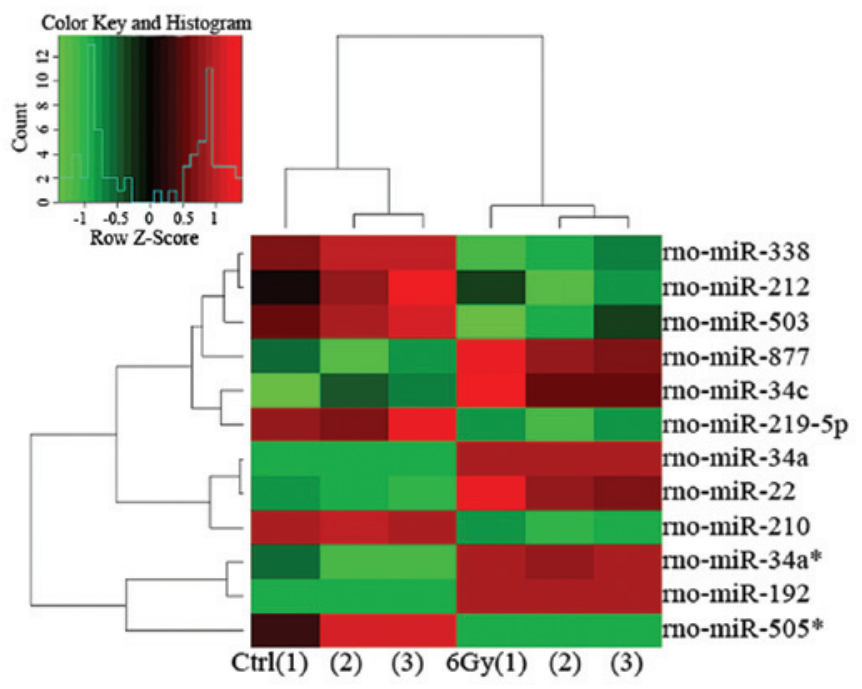

Figure 2. Heatmap of the 12 significantly altered miRNAs subsequent to exposure to 6 Gy radiation for 5 days. These 12 miRNAs were selected according to the fold changes and microarray repeatability. The upregulated miRNAs are depicted in shades of red and downregulated miRNAs are depicted in shades of green. The black blocks represent unchanged expression of the miRNAs. There were three samples $(n=3)$ in each condition.

the alterations in miRNA expression involved in radiation injury to RGCs and their detailed functions remain to be fully elucidated. In the current study, different doses of ionizing radiation were applied to induce radiation injury in cultured RGC-5 cells. Alterations in the miRNA expression levels were evaluated following radiation injury, which may aid in understanding the molecular mechanisms of radiation-induced retinopathy and RION.
The data of the present study revealed that exposure to 6 Gy and 8 Gy doses of X-ray radiation resulted in radiation injury in RGC-5 cells. The viability of RGC- 5 cells was significantly reduced, which was in accordance with previously reported results $(20,21)$. The microarray assay demonstrated that there was a total of 37 miRNAs that were statistically altered in the radiation-treated RGC-5 cells. It is known that when using microarray to detect differentially expressed miRNA, multiple tests are required to ensure that the results are stable and repeatable (22). A minimum of 3 repeats of each experiment were performed in the current study, following which 12 significantly altered miRNAs were selected according to the fold change and microarray repeatability, including 6 upregulated miRNAs (miR-192, -34a*, -877, -34c, -34a and -22) and 6 downregulated miRNAs (miR-212, -338, -219-5p, -503, -210 and $-505^{*}$ ). Microarray technology is a powerful tool used to detect whether a set of miRNAs are differentially expressed. However, microarray profiling of microRNA expression raises a number of analytical challenges that must be addressed in order to obtain reliable results, and RT-qPCR analysis has been commonly used for further validation (23). In the current study, the data from RT-qPCR analysis were consistent with that from microarray analysis, indicating that the expression levels of these miRNAs were regulated in response to the radiation-induced injury in RGC-5 cells.

Ocular damage is a common side effect of radiotherapy, such as dry eye, epiphora, ectropion, scleral necrosis, cataract, glaucoma, optic neuropathy and retinopathy. Radiation-induced retinopathy and RION are the most serious complications in terms of visual damage resulting from radiation therapy for tumors adjacent to the retina and optic nerves (24). The pathogenesis of radiation-induced retinopathy and RION include vascular occlusion, injury to the retinal ganglion cells and axons or a combination of these effects (25). In a dog model of radiation-induced retinal injury, retinal degeneration with swelling and loss of the ganglion cells was observed following radiation treatment for 1 year (26). In humans, retinopathy commonly appears 6 months-3 years following radiation therapy, characterized by irreversible ganglion and glial cell necrosis and vasculitis (27). At present, the molecular mechanisms of ionizing radiation-induced ganglion cell injury remain to be fully elucidated. The data of the current study indicated that there were 12 miRNAs involved in the process of radiation-induced injury in RGC-5 cells. According to the reports, the function of these miRNAs and their regulated target genes are predominantly associated with the cell cycle and proliferation (miR-192, -22, -212 and -219-5p) (28-31), metabolism (miR-34a and -34c) (32) and apoptosis and anti-apoptosis (miR-192, -34a, -34c, -338 and -210) (28,33,34). For example, miR-192 is able to regulate p53 expression to induce tubular epithelial cell growth cycle arrest and DNA damage following kidney acute injury (28). miR-34a and miR-34c have been demonstrated to be downstream effectors of p53-mediated human fibroblast apoptosis and senescence (34). Further investigation of these miRNAs may aid in the understanding of the pathogenesis and development of radiation-induced retinopathy and RION.

Although numerous treatments have been suggested, management of radiation-induced retinopathy and RION remains controversial (9). At present, clinical therapeutic 
options include the use of corticosteroids, anticoagulants, antiaggregants and hyperbaric oxygen (HBO). A retrospective literature review identified there was no favorable effect of either corticosteroids or anticoagulation (35) and cases of radiation damage and visual loss have been reported to develop despite anticoagulation therapies (36). A previous study reported that HBO was a beneficial therapy for patients with radiation-induced retinopathy and RION (37). However, it has been suggested that HBO therapy is only effective in certain cases when patients were treated with $\mathrm{HBO}$ as early as possible following the onset of visual loss (37). According to characteristics of radiation-induced retinopathy and RION, the onset of visual acuity loss may be as short as 3 months or as long as 8 years following radiation exposure (38). Once radiotherapy has induced retinal complications, visual damage has been reported to progress quickly and be difficult to control (9). Early diagnosis is extremely important for the prevention and treatment of radiation-induced retinopathy $(10,11)$. However, an effective method to monitor the early stages of radiation-induced retinopathy and RION remains to be developed.

A previous study demonstrated that miRNA expression is significantly different between pathological tissues and normal tissues (39). Understanding of the potential use of miRNA expression levels as biomarkers for the early detection of numerous diseases is on the increase (40). In the current study, 12 miRNAs, which were all sensitive to ionizing radiation and were differentially expressed between the experimental and control groups, were screened using microarray analysis in RGC-5 cells following radiation exposure. Due to the fact that it remains a challenge to detect and treat radiation-induced retinopathy and RION at an early stage, these 12 miRNAs are suggested as novel potential biomarkers for diagnosis, prediction of prognosis and therapeutic management.

Although the results of the current study are promising, there are several limitations. Firstly, a clear drawback of the present study is that it is an in vitro experiment. The environment of cells in vivo is different to cells cultured in vitro, however, it is easy to control the experimental conditions using cultured cells and the results are often more accurate. In addition, it has been identified that the majority of cells release pathogenic miRNAs into the extracellular environment of cells, where they are protected by RNase and remain relatively stable (41). Expression levels of miRNAs associated with diseases in biological fluids have been suggested as promising biomarkers for diagnosis and therapeutic monitoring (12-15). Concerning the 12 miRNAs screened in the current study, it remains unclear whether they are released by the retinal ganglion cells into the extracellular environment following radiation exposure. Thus, further studies are required to investigate the possibilities of the 12 miRNAs as potential biomarkers for radiation-induced retinopathy and RION.

In conclusion, the current study suggested that cultured RGC-5 cells were sensitive to ionizing radiation injury. The cell viability was significantly reduced following radiation exposure, accompanied by alterations in miRNA expression. These altered miRNAs may serve an important role in the mechanisms of radiation-induced retinopathy and RION, and may be used as potential biomarkers for the early detection of retinal and optic nerve radiation injury.

\section{Acknowledgements}

The current study was financially supported by research grants from the National Natural Science Foundation of China (grant nos. 81372930 and 30700443) and the Zhejiang Provincial Natural Science Foundation of China (grant nos. LY12H12008 and LQ15H120001).

\section{References}

1. Bartel DP: MicroRNAs: Genomics, biogenesis, mechanism, and function. Cell 116: 281-297, 2004.

2. Graves P and Zeng Y: Biogenesis of mammalian microRNAs: A global view. Genomics Proteomics Bioinformatics 10: 239-245, 2012.

3. Huang Y, Shen XJ, Zou Q and Zhao QL: Biological functions of microRNAs. Bioorg Khim 36: 747-752, 2010.

4. Sundermeier TR and Palczewski K: The physiological impact of microRNA gene regulation in the retina. Cell Mol Life Sci 69: 2739-2750, 2012.

5. Karali M, Peluso I, Marigo V and Banfi S: Identification and characterization of microRNAs expressed in the mouse eye. Invest Ophthalmol Vis Sci 8: 509-515, 2007.

6. Roden D, Bosley TM, Fowble B, Clark J, Savino PJ, Sergott RC and Schatz NJ: Delayed radiation injury to the retrobulbar optic nerves and chiasm. Clinical syndrome and treatment with hyperbaric oxygen and corticosteroids. Ophthalmology 97: 346-351, 1990.

7. Chaudhry MA, Omaruddin RA, Kreger B, de Toledo SM and Azzam EL: MicroRNA responses to chronic or acute exposures to low dose ionizing radiation. Mol Biol Rep 39: 7549-7558, 2012.

8. Templin T, Paul S, Amundson SA, Young EF, Barker CA, Wolden SL and Smilenov LB: Radiation-induced micro-RNA expression changes in peripheral blood cells of radiotherapy patients. Int J Radiat Oncol Biol Phys 80: 549-557, 2011.

9. Lee MS and Borruat FX: Should patients with radiation-induced optic neuropathy receive any treatment? J Neuroophthalmol 31: 83-88, 2011.

10. Levy RL and Miller NR: Hyperbaric oxygen therapy for radiation-induced optic neuropathy. Ann Acad Med Singapore 35: 151-157, 2006.

11. Danesh-Meyer HV: Radiation-induced optic neuropathy. J Clin Neurosci 15: 95-100, 2008.

12. Mo MH, Chen L, Fu Y, Wang W and Fu SW: Cell-free circulating miRNA biomarkers in cancer. J Cancer 3: 432-448, 2012.

13. Li JY, Yong TY, Michael MZ and Gleadle JM: Review: The role of microRNAs in kidney disease. Nephrology (Carlton) 15: 599-608, 2010.

14. Hulsmans M and Holvoet P: MicroRNAs as early biomarkers in obesity and related metabolic and cardiovascular diseases. Curr Pharm Des 19: 5704-5717, 2013.

15. Zhao Z, Zhao Q, Warrick J, Lockwood CM, Woodworth A, Moley KH and Gronowski AM: Circulating microRNA miR-323-3p as a biomarker of ectopic pregnancy. Clin Chem 58: 896-905, 2012.

16. Schöler N, Langer C, Döhner H, Buske C and Kuchenbauer F: Serum microRNAs as a novel class of biomarkers: A comprehensive review of the literature. Exp Hematol 38: 1126-1130, 2010.

17. Kroh EM, Parkin RK, Mitchell PS and Tewari M: Analysis of circulating microRNA biomarkers in plasma and serum using quantitative reverse transcription-PCR (qRT-PCR). Methods 50: 298-301, 2010.

18. Pinter R and Hindges R: Perturbations of microRNA function in mouse dicer mutants produce retinal defects and lead to aberrant axon pathfinding at the optic chiasm. PLoS One 5: e10021, 2010.

19. Marler KJ, Suetterlin P, Dopplapudi A, Rubikaite A, Adnan J, Maiorano NA, Lowe AS, Thompson ID, Pathania M, Bordey A, et al: BDNF promotes axon branching of retinal ganglion cells via miRNA-132 and p250GAP. J Neurosci 34: 969-979, 2014.

20. Lulli M, Witort E, Papucci L, Torre E, Schiavone N, Dal Monte M and Capaccioli S: Coenzyme Q10 protects retinal cells from apoptosis induced by radiation in vitro and in vivo. J Radiat Res 53: 695-703, 2012.

21. Balaiya S, Malyapa R, Hsi W, Murthy RK and Chalam KV: Evaluation of proton beam radiation sensitivity of proliferating choroidal endothelial and retinal ganglion cells with clonogenic assay. Cutan Ocul Toxicol 31: 14-19, 2012. 
22. Wang B and Xi Y: Challenges for microRNA microarray data analysis. Microarrays (Basel) 2, 2013.

23. Sarkar D, Parkin R, Wyman S, Bendoraite A, Sather C, Delrow J, Godwin AK, Drescher C, Huber W, Gentleman R, et al: Quality assessment and data analysis for microRNA expression arrays. Nucleic Acids Res 37: e17, 2009.

24. Finger PT: Tumour location affects the incidence of cataract and retinopathy after ophthalmic plaque radiation therapy. Br J Ophthalmol 84: 1068-1070, 2000 .

25. Wolfensberger TJ,Zwingli M, Egger E, Schnyder P and Zografos L: Subclinical experimental optic neuropathy after accelerated proton beam irradiation. Ophthalmologica 216: 420-425, 2002.

26. Pinard CL, Mutsaers AJ, Mayer MN and Woods JP Retrospective study and review of ocular radiation side effects following external-beam Cobalt- 60 radiation therapy in 37 dogs and 12 cats. Can Vet J 53: 1301-1307, 2012.

27. Gupta A, Dhawahir-Scala F, Smith A, Young L and Charles S: Radiation retinopathy: Case report and review. BMC Ophthalmol 7: 6, 2007.

28. Hong JP, Li XM, Li MX and Zheng FL: VEGF suppresses epithelial-mesenchymal transition by inhibiting the expression of Smad3 and miR-192, a Smad3-dependent microRNA. Int J Mol Med 31: 1436-1442, 2013.

29. Li S, Hu R, Wang C, Guo F, Li X and Wang S: miR-22 inhibits proliferation and invasion in estrogen receptor $\alpha$-positive endometrial endometrioid carcinomas cells. Mol Med Rep 9: 2393-2399, 2014.

30. Tognini P and Pizzorusso T: MicroRNA212/132 family: Molecular transducer of neuronal function and plasticity. Int J Biochem Cell Biol 44: 6-10, 2012.

31. Huang N, Lin J, Ruan J, Su N, Qing R, Liu F, He B, Lv C, Zheng D and Luo R: MiR-219-5p inhibits hepatocellular carcinoma cell proliferation by targeting glypican-3. FEBS Lett 586: 884-891, 2012.

32. Liu K, Huang J, Xie M, Yu Y, Zhu S, Kang R, Cao L, Tang D and Duan X: MIR34A regulates autophagy and apoptosis by targeting HMGB1 in the retinoblastoma cell. Autophagy 10 : $442-452,2014$
33. Ragusa M, Majorana A, Banelli B, Barbagallo D, Statello L, Casciano I, Guglielmino MR, Duro LR, Scalia M, Magro G, et al: MIR152, MIR200B, and MIR338, human positional and functional neuroblastoma candidates, are involved in neuroblast differentiation and apoptosis. J Mol Med (Berl) 88: 1041-1053, 2010.

34. Kumamoto K, Spillare EA, Fujita K, Horikawa I, Yamashita T, Appella E, Nagashima M, Takenoshita S, Yokota J and Harris CC: Nutlin-3a activates p53 to both down-regulate inhibitor of growth 2 and up-regulate mir-34a, mir-34b, and mir-34c expression and induce senescence. Cancer Res 68: 3193-3203, 2008.

35. Guy J and Schatz NJ: Hyperbaric oxygen in the treatment of radiation induced optic neuropathy. Ophthalmology 93: 1083-1088, 1986.

36. Danesh Meyer HV, Savino PJ and Sergott RC: Visual loss despite anticoagulation in radiation-induced optic neuropathy. Clin Experiment Ophthalmol 32: 333-335, 2004

37. Levy RL and Miller NR: Hyperbaric oxygen therapy for radiation induced optic neuropathy. Ann Acad Med Singapore 35: 151-157, 2006.

38. Demizu Y, Murakami M, Miyawaki D, Niwa Y, Akaqi T, Sasaki R, Terashima K, Suqa D, Kamae I and Hishikawa Y: Analysis of vision loss caused by radiation-induced optic neuropathy after particle therapy for head-and-neck and skull-base tumors adjacent to optic nerves. Int J Radiat Oncol Biol Phys 75: 1487-1492, 2009.

39. Alvarez-Garcia I and Miska EA: MicroRNA functions in animal development and human disease. Development 132: 4653-4662, 2005.

40. Kress M, Hüttenhofer A, Landry M, Kuner R, Favereaux A, Greenberg D, Bednarik J, Heppenstall P and Kronenberg F: MicroRNAs in nociceptive circuits as predictors of future clinical applications. Front Mol Neurosci 6: 33, 2013.

41. Cheng L, Sharples RA, Scicluna BJ and Hill AF: Exosomes provide a protective and enriched source of miRNA for biomarker profiling compared to intracellular and cell-free blood. J Extracell Vesicles 26: 3, 2014. 